\title{
Metallothioneins mediated intracellular copper homeostasis in ectomycorrhizal fungus Suillus indicus
}

\author{
Shikha Khullar, Anuja Sharma, Radhika Agnihotri and M. Sudhakara Reddy* \\ Department of Biotechnology, Thapar Institute of Engineering \& Technology, Patiala, 147004, Punjab, India \\ *Corresponding author email: msreddy@thapar.edu
}

(Submitted on October 27, 2019; Accepted on December 20,2019)

\begin{abstract}
Ectomycorrhizal fungi (ECM) are known to protect the host plant from heavy metal stress. But the information on molecular mechanisms involved in this process is still ambiguous. The present study intends on providing insight into the Cu detoxification mechanism in ECM fungus Suillus indicus, a new species isolated from north western Himalayas. Two metallothionein genes SuiMT1 and SuiMT2, were isolated from the $S$. indicus cDNA and characterized for their potential role in $\mathrm{Cu}$-detoxification and homeostasis. The response of these genes to the extracellular concentrations of copper was studied by qPCR analysis. Both genes were actively induced under exogenous Cu stress, thus can be classified as $\mathrm{Cu}$-thioneins. Further, the functional complementation of these genes in the Cu sensitive yeast mutant cup ${ }^{\Delta}$, successfully restored their wild type phenotype of $\mathrm{Cu}$ tolerance. This shows that both $\mathrm{SuiMT1}$ and $\mathrm{SuiMT2}$ plays an important role in Cu detoxification and homeostasis in ECM fungus $S$. indicus.
\end{abstract}

KEYWORDS: Ectomycorrhizal fungi, Suillus indicus, metallothionein, copper, metal homeostasis, yeast complementation, qPCR

\section{INTRODUCTION}

Ectomycorrhizal fungi (ECM) forms a mutualistic association with the plant roots thus providing them with various nutrients and protecting them from various biotic and abiotic stresses. It is very well known that these ECM fungi protect plants from heavy metals, drought, salinity, pests and pathogens and extreme environments, but the exact mechanisms involved are still inexplicit. There are different mechanisms in ECM fungi to protect itself from heavy metals like, cell wall binding (Bano et al., 2018), extracellular chelation, metal efflux (Ruytinx et al., 2013; Sacký et al., 2016; Benes et al., 2018) or their intracellular chelation through various metal binding ligands like metallothioneins and glutathione (Osobova et al., 2011; Reddy et al., 2016; Kalsotra et al., 2018; Khullar and Reddy, 2018, 2019a,b).

Metallothioneins(MTs) are a superfamily of low molecular weight proteins, ubiquitous and polyphyletic, that coordinates heavy metal ions by establishing the metalthiolate bond through their highly abundant cysteine residues (Calvo et al., 2017). These metalloproteins are then accumulated into the vacuoles and later released as metallic complex (Bellion et al., 2006). They are usually characterized by highly conserved $\mathrm{C}-\mathrm{X}-\mathrm{C}, \mathrm{C}-\mathrm{X}-\mathrm{X}-\mathrm{C}, \mathrm{C}-\mathrm{X}-\mathrm{Y}-\mathrm{C}$ motifs (Ramesh et al., 2009; Reddy et al., 2014; Hložková et al., 2016; Zahid et al., 2016; Nguyen et al., 2017; Khullar and Reddy, 2018). It has numerous physiological and biological functions such as detoxification of toxic heavy metals, metal homeostasis, free radical scavenging and protection against oxidative stress. MTs are pervasive and present in both prokaryotes and eukaryotes. Different MTs exhibit different metal preferences under different conditions. Palacios et al. (2011) classified MTs into two categories as $\mathrm{Cu}$-thioneins and $\mathrm{Zn} / \mathrm{Cd}$-thioneins as per their metal binding preferences (Palacios et al., 2011). Cu-thioneins form homometallic $\mathrm{Cu}-$ MT complexes when exposed to $\mathrm{Cu}$ and $\mathrm{Zn} / \mathrm{Cd}$ thioneins form homometallic complexes when exposed to $\mathrm{Zn}$ or $\mathrm{Cd}$, respectively with high degree of folding. However, when exposed to other metals they form heteronuclear complexes with a lower degree of folding and high thiol oxidation resulting in disulfide formation (Palacios et al., 2011). Inspite of all the information, MTs biological structure and their role in various physiological processes in diverse living systems are still a matter of debate. Although many studies have reported diverse MT genes isolated from prokaryotic bacteria (Solioz, 2018), mammals (Atrián-Blasco et al., 2017; Drozd et al., 2018), plants (Huang et al., 2018; Imam and Blindauer, 2018), animals (Li et al., 2016) and fungal species (IturbeEspinoza et al., 2016) etc, but not much has been reported in ectomycorrhizal systems. The metal binding properties of metallothioneins isolated from same organism are different for different metals and host species. Three MT isoforms isolated from Amanita strobiliformis (AsMT1, AsMT2, AsMT3) responded differently to different heavy metals. When subjected to different metals, AsMTl was induced by copper and silver, whereas $A s M T 2$ was induced by cadmium and AsMT3 was induced by zinc (Hložková et al., 2016). Similar observations were made in L. bicolor, where the two isoforms $L b M T 1$ and $L b M T 2$ were differentially induced by cadmium and copper, respectively (Reddy et al., 2014). Similar results have also been reported in Hebeloma mesophaeum, Hebeloma cylindrosporum, Pisolithus albus, Paxillus involutus, Suillus luteus and Suillus himalayensis (Bellion et al., 2007; Ramesh et al., 2009; Sácký et al., 2014; Reddy et al., 2016; Nguyen et al., 2017; Kalsotra et al., 2018).

Recently, a new species 'Suillus indicus' was collected from the conifer forests of the north western Himalayas, India, forming ectomycorrhizal association with Pinus wallichiana and Cedrus deodara (Verma and Reddy, 2015). The fungus was shown to promote the growth of Pinus wallichiana seedlings in the nursery (data not provided). Observing the impact of Suillus indicus, we considered identifying its potential in metal detoxification. For this, two MTs (SuiMT1 and SuiMT2) were identified from the $S$. indicus cDNA, by designing the primers from the EST transcripts of Suillus luteus (Ruytinx et al., 2011). Further, the role of both MTs in copper detoxification was studied using real time PCR and yeast functional complementation.

\section{MATERIALS AND METHODS}

Organisms, culture media and culture conditions: The ectomycorrhizal fungus Suillus indicus, isolated from the north western region of Himalayas was maintained on malt 
extract medium (2\%) with pH 5.5 at $25^{\circ} \mathrm{C}$ in the dark (Verma and Reddy, 2015). The tolerance of $S$. indicus to copper was studied by growing the mycelium in MMN broth supplemented with increasing concentrations of copper $(0$, $100,200,300$ and $400 \mu \mathrm{M}$ as $\left.\mathrm{CuSO}_{4} .5 \mathrm{H}_{2} \mathrm{O}\right)$ at $25^{\circ} \mathrm{C}$ in dark for 21 days. After 21 days, the mycelium was harvested under each stress and washed with EDTA water followed by two washing with distilled water. The washed mycelium was then dried at $48^{\circ} \mathrm{C}$ and the dry weight was recorded as the effect of $\mathrm{Cu}$ on mycelial growth. Further, the intracellular metal accumulation was determined using atomic absorption spectroscopy.

The E. coli $\mathrm{DH} 10 \beta$ cells were used to maintain and propagate various plasmids according to standard protocols (Sambrook and Russell, 2001). The copper sensitive Saccharomyces cerevisiae strain used for yeast complementation assays-

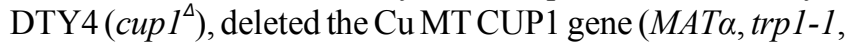
leu2-3, leu2-112, gal1, his-, ura3-50, cupl ${ }^{4}:$ URA3+) (Hamer et al., 1985) derived from DTY3 wild-type strain (MATo, trp1-1,leu2-3, leu2-112, gal1, his , ura3-50, URA3').

RNA isolation, cDNA synthesis and gene amplification: Mycelial discs (7mm diam.) of Suillus indicus were cultured on MMN-agar plates overlaid with cellophane sheets for 15 days at $25^{\circ} \mathrm{C}$ in the dark. After 15 days, the mycelium was transferred along with cellophane sheet to $\mathrm{MMN}$ medium supplemented with different concentrations of copper $(0,100$, 200, 300 and $400 \mu \mathrm{M}$ as $\mathrm{CuSO}_{4} \cdot 5 \mathrm{H}_{2} \mathrm{O}$ ) and incubated for 48 hours at $25^{\circ} \mathrm{C}$ in the dark. The mycelium was then scrapped and crushed in liquid nitrogen. The total RNA was extracted from the crushed mycelia using the QiAzol lysis reagent (QIAGEN, Germany). The integrity of the isolated RNA was checked by formaldehyde agarose gel electrophoresis and RNA concentration was determined by measuring the absorbance at $260 \mathrm{~nm}$ on nanodrop. cDNAs were synthesized from approximately $5 \mu \mathrm{g}$ of total RNA using "The revert aid first strand cDNA synthetic kit" (Thermo Fisher Scientific, U.S) as per manufacturer's instructions. Amplification of SuiMT1 and SuiMT2 genes were performed with the primers (Table 1) designed from EST sequences (GR975901, GR975896, GR975714, GR975715, GR975716) of putative MTs of $S$. luteus.

PCR reactions were carried out in $25 \mu \mathrm{L}$ reaction containing 1x PCR buffer, $1.5 \mathrm{mM} \mathrm{MgCl}_{2}, 200 \mu \mathrm{M}$ dNTP mix, $1 \mu \mathrm{L}$ of forward and reverse primer $(10 \mu \mathrm{M})$ (Table 1), $1.5 \mathrm{U}$ Taq polymerase (Sigma-Aldrich), $1 \mu \mathrm{L}$ template and nuclease free water. Starting with an initial denaturation at $94^{\circ} \mathrm{C}$ for $3 \mathrm{~min}$, SuiMT1 and SuiMT2 fragments were amplified for 35 cycles comprising $1 \mathrm{~min}$ at $94^{\circ} \mathrm{C}, 1 \mathrm{~min}$ at $55^{\circ} \mathrm{C}$ and $1 \mathrm{~min}$ at $72^{\circ} \mathrm{C}$ followed by final extension at $72^{\circ} \mathrm{C}$ for $8 \mathrm{~min}$. PCR products were run on $1.5 \%(\mathrm{w} / \mathrm{v})$ agarose gel and visualized by ethidium bromide staining. The amplified product was purified by using GeneJet PCR purification kit (Thermo scientific) and ligated in pMD20 vector (TA cloning kit, Takara). The ligated products were transformed into E.coli $\mathrm{DH} 10 \beta \mathrm{CaCl}_{2}$ competent cells by heat shock method (Singh et al., 2010) and plated on Luria Agar plates supplemented with ampicillin, IPTG and X-gal for blue-white screening. The white colonies were randomly selected and verified by colony
PCR. The positive colonies so obtained were sequenced and analyzed using various bioinformatic tools.

Table 1: PCR primers designed for the amplification of SuiMT1 and SuiMT2 gene of Suillus indicus and for qPCR analysis

\begin{tabular}{lll}
\hline Gene Name & Primer & Sequence \\
\hline SuiMT1 & SuiMTIF & 5'-CGGGATCCATGTCCACCGCTACTGAAGTC -3' \\
& SuiMTIR & 5'-CCGGAATTCTCAACATTTGCACTCTCCAGG-3' \\
SuimT2 & SuiMT2F & 5'-CGGGATCCATGTCCACCGCTACTGAAGTC -3' \\
& SuiMT2R & 5'-CCGGAATTCTCAATCAACATTGCACTCTCCAG -3' \\
a-actin & SactF & 5'-GTATTGCCGACCGTATGCAG -3' \\
& SactR & 5'-GGAGCGACGATCTTGACCTA -3', \\
\multirow{3}{*}{-tubulin } & StubF & 5'-GTGGACTCTAGCGGACCTAC-3', \\
& StubR & 5'-CCCTGAGTCGCTAGTGAAGT-3' \\
\hline
\end{tabular}

Underlined sequences are Bam $\mathrm{H} 1$ and $E c o \mathrm{R} 1$ sites

Bioinformatic analysis: The open reading frame (ORF) of both SuiMT1 and SuiMT2 sequences was identified using ORF finder. Both the sequences were then subjected to BLASTp analysis, to identify the homologous sequences. The homologous sequences so obtained were aligned using Multalin (http://multalin.toulouse.inra.fr/multalin/) so as to identify the conserved C-X-C motifs. Further the molecular weight and $\mathrm{pI}$ of the predicted proteins were calculated using Expasy tool (https://www.expasy.org/).

Expression analysis of SuiMT1 and SuiMT2 (qRT-PCR): The two weeks culture of $S$. indicus grown on MMN agar plates overlaid with cellophane sheets was stressed with increasing concentrations of $\mathrm{Cu}(0,100,200,300$ and 400 $\mu \mathrm{M})$ for 48 hours at $25^{\circ} \mathrm{C}$. The stressed mycelium was then scrapped from the cellophane sheets and grounded in liquid nitrogen. From each stressed sample total RNA was isolated and cDNA was synthesized as per the protocols mentioned previously. Gene expression analysis of SuiMT1 and SuiMT2 in the mycelium was performed in mastercycler $\AA$ ep realplex real-time PCR system (Eppendorf AG, Hamburg, Germany) using SYBR ${ }^{\circledR}$ Green JumpStart ${ }^{\mathrm{TM}}$ TaqReady Mix $^{\mathrm{TM}}$ (Sigma Aldrich) under the conditions recommended by the manufacturer. The reaction was performed in total volume of $25 \mu \mathrm{L}$, consisting of $12.5 \mu \mathrm{L}$ master mix, $1 \mu \mathrm{L}$ each of forward and reverse primer (Table 1), $0.75 \mu \mathrm{L}$ cDNA template and $9.75 \mu \mathrm{L} \mathrm{H}_{2} \mathrm{O}$. The cycling program used for qPCR was as follow: $95^{\circ} \mathrm{C}$ for $2 \mathrm{~min}$ ( 1 cycle), $95^{\circ} \mathrm{C}$ for $15 \mathrm{~s}, 55^{\circ} \mathrm{C}$ for $15 \mathrm{~s}$ and $68^{\circ} \mathrm{C}$ for $20 \mathrm{~s}$ ( 40 cycles). The relative quantification of gene expression between samples was calculated using the comparative threshold (Ct) method (Heid et al., 1996). $\alpha$ actin (SbAct) (Accession: AF155930) and $\beta$-tubulin (Accession: AY112730) of Suillus bovinus were used as reference genes. Since the NormFinder observed minimum stability value for $\alpha$-actin, it was used for the comparative analysis. The amplification efficiency of gene was calculated by the equation $\mathrm{E}=\left[10^{(-1 / \text { slope })}\right]$. The $\mathrm{E}$ value so obtained (1.25) was used to calculate $C_{t 1}$ value, where $C_{t 1}=C_{t e} x$ $[\log (1+E) / \log 2]$. The $C_{t 1}$ value was calculated for each sample and then the comparative expression level of the genes was given by the formula $2^{-\Delta \Delta C \mathrm{CT}}$ (Livak and Schmittgen, 2001) where $\Delta \Delta \mathrm{CT}$ was calculated by subtracting the baseline's $\Delta \mathrm{CT}$ to the sample's $\Delta \mathrm{CT}$ and where the baseline represents the expression level of the control. All measurements were performed on independent biological samples from three replicate experiments in three technical replicates. 
Cloning of MT genes: Metallothionein genes SuiMT1 and SuiMT2 were amplified from the cDNA of Suillus indicus (as described previously). The genes so obtained, along with the yeast expression vector pFL61 were subjected to double digestion with restriction endonucleases EcoR 1 and $B a m \mathrm{H} 1$ for 4 hours at $37^{\circ} \mathrm{C}$. The digested genes and plasmid were run on $1 \%$ agarose gel and the required bands were excised using Thermo Scientific GeneJet Gel Extraction kit as per the prescribed protocol. The digested genes were further ligated into pFL61 using T4 DNA ligase. Further, the ligated product (pFL61+SuiMT1 and pFL61+SuiMT2) was transformed into E.coli DH5 $\alpha$ cells and the positive clones were screened on LA ampicillin plates by colony PCR followed by plasmid isolation. The plasmid so obtained (pFL61, pFL61+SuiMT1 and pFL61+SuiMT2) were further transformed into copper sensitive yeast mutant $\left(\right.$ cupl $\left.^{\Delta}\right)$ using lithium acetate method (Stearns et al., 1990) and the positive clones were selected by their tendency to grow on complete synthetic medium without uracil (SD-Ura). The positive clones so obtained were further used for yeast functional complementation studies against copper stress.

Yeast functional complementation assays: For studying the functional complementation of SuiMTland SuiMT2 genes in

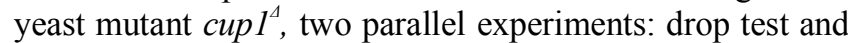
growth kinetics were performed. For Drop test, cultures of

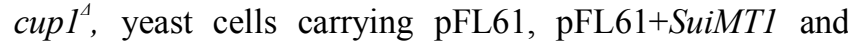
pFL61+SuiMT2 were grown in SD-Ura media for 24 hours at $30^{\circ} \mathrm{C}$ and $220 \mathrm{rpm}$. After 24 hours, optical density of all the three cultures was adjusted to $\mathrm{OD}_{600}=1.0$. The cultures were serially diluted $\left(10^{-1}, 10^{-2}\right.$ and $\left.10^{-3}\right)$ and $5 \mu \mathrm{L}$ of each dilution was spotted on SD-Ura plates supplemented with and without $150 \mathrm{mM} \mathrm{CuSO}_{4}$. Plates were incubated for 3 days at $30^{\circ} \mathrm{C}$ and photographed. In a parallel experiment, to analyze the growth kinetics of the SuiMT1 and SuiMT2 transformants, $20 \mathrm{~mL}$ of fresh SD-Ura media were inoculated with mid-exponential pre-cultures of cup $^{4}$ containing pFL61, pFL61+SuiMT1 and pFL61+SuiMT2 to attain a starting optical density of 0.02 at
$600 \mathrm{~nm}$. All the cultures at O.D 0.02 were grown at $30^{\circ} \mathrm{C}$ in a rotary shaker $(220 \mathrm{rpm})$ for 5 hours followed by addition of $150 \mu \mathrm{M} \mathrm{CuSO}_{4}$ to transformants. The cells were allowed to grow for next 48 hours, where the growth of each culture was monitored by taking the $\mathrm{O}^{-D_{600}}$ at every 3 hours interval. The data obtained was plotted as a graph and analysed by ANOVA. The means were compared with Tukey's test at $\mathrm{P}<0.05$. All the analysis was performed by using Graph Pad Prism 5.1 software.

\section{RESULTS}

Effect of copper on the growth and metal uptake of $S$. indicus: When subjected to different concentrations of copper $(0,100,200,300,400 \mu \mathrm{M})$, the growth of $S$. indicus was adversely affected. Approximately $16 \mathrm{mg}$ of fungal biomass was procured after 21 days when grown in $50 \mathrm{~mL}$ unstressed MMN broth. However, the fungal biomass declined when the same broth was supplemented with different concentrations of copper. The half minimum inhibitory concentration of $\mathrm{Cu}\left(\mathrm{IC}_{50}\right)$ was observed at $170 \mu \mathrm{M}$, where the fungal biomass was reduced to $50 \%$ (Fig. 1a). However, the $\mathrm{Cu}$ uptake by the mycelium was found to increase as a function of external $\mathrm{Cu}$ concentration. At 100 $\mu \mathrm{M}$ of external $\mathrm{Cu}$, approximately $0.75 \mu \mathrm{g}$ of $\mathrm{Cu}$ was uptaken per mg of fungal dry weight. However, at $400 \mu \mathrm{M}$ of external $\mathrm{Cu}$, the metal uptake was found to elevate up to $1.22 \mu \mathrm{g} / \mathrm{mg}$ of fungus (Fig. 1b).

Sequence analysis of SuiMT1 and SuiMT2: Five transcripts were identified from the putative MTs from the EST library of Suillus luteus (Ruytinx et al., 2011). Amongst the five transcripts, two distinct putative MTs were opted and their specific primers were synthesized (Table 1). Both MTs were amplified from the Suillus indicus cDNA and sequenced. The sequence analysis of both SuiMT1 and SuiMT2, revealed that both the sequences showed high homology with metallothionein genes reported from various basidiomycetes. Further, the multiple sequence analysis of the homologous
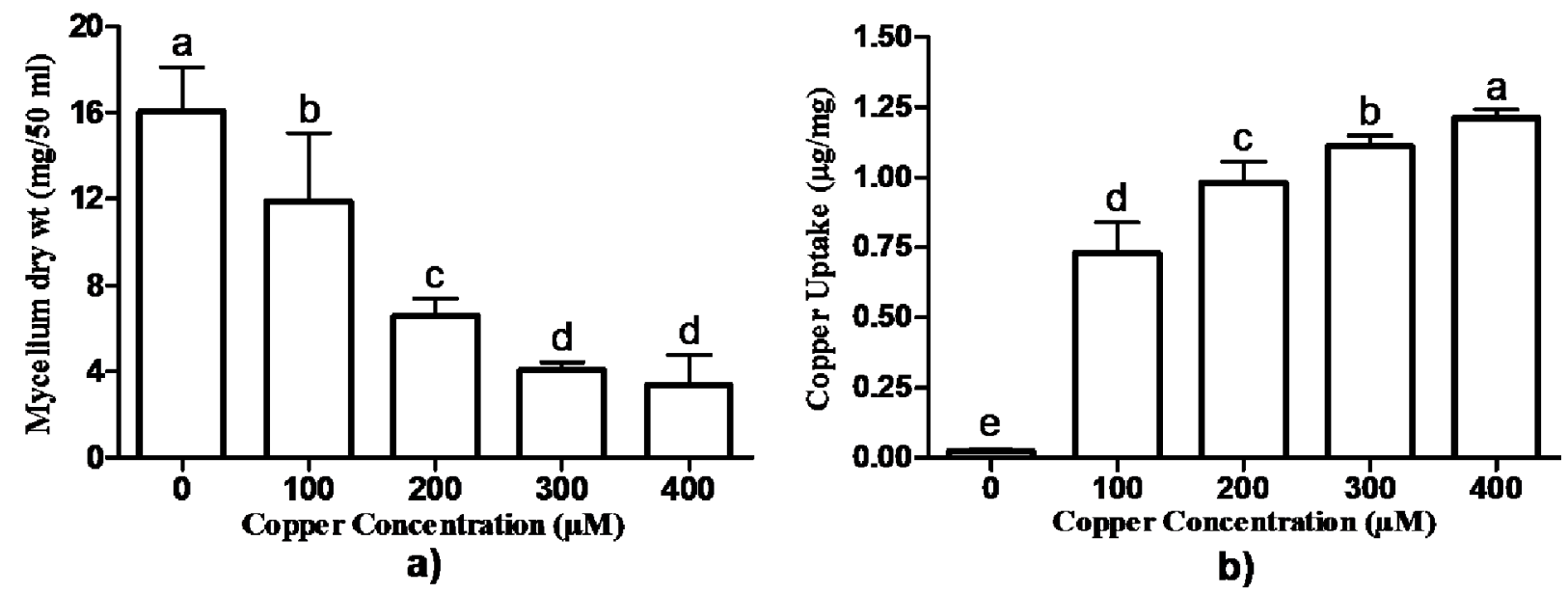

Fig. 1 Effect of different concentrations of copper on a) mycelium growth and b) metal uptake in ectomycorrhizal fungus Suillus indicus, when grown on $\mathrm{MMN}$ medium supplemented with $\mathrm{CuSO}_{4}$. 


\begin{tabular}{|c|c|c|}
\hline SuiMT1 & MSTATEVLV-SNNN & 34 \\
\hline SuiMT2 & MSTATEVLV-SNNNCGSLIST & 35 \\
\hline ShMT1 & MSTATEVPV-SNN & 34 \\
\hline PaMT & MQSVNAVLVNNNG & 35 \\
\hline SiMT1 & MISETIVPV-N- & 33 \\
\hline IbMT1 & MISTINVPV-S-Q & 33 \\
\hline AsMT2 & MQSESQSLV-SFA & 34 \\
\hline RaMT & MSPVIQNPV-NEHHC & 34 \\
\hline PIMT1 & MNTITSVPV-NFN & 34 \\
\hline
\end{tabular}

Fig. 2. Multiple sequence alignment of SuiMT1 and SuiMT2 genes with their homologous sequences retrieved through BLASTp analysis. The conserved C-x-C motifs amongst metallothioneins from different basidiomycetes i.e Suillus himalayensis (ShMT1; AUS94321), Pisolithus albus (PaMT; AJO67962), Serendipita indica (SiMT1; ACT83730), Laccaria bicolor (LbMT1; AHI43933), Amanita strobiliformis (AsMT2; AG004615), Russula atropurpurea (RaMT; AHA31882), Paxillus involutus (PiMT1; AAS19463) were highlighted in blue.

sequences highlighted three conserved $\mathrm{C}-\mathrm{x}-\mathrm{C}$ motifs, which are the main characteristic to metallothioneins (Fig. 2). SuiMTlconsists of 105 bp ORF coding for 34 amino acids with a predicted molecular weight of $3.4 \mathrm{kDa}$ and $\mathrm{pI} 5.9$. SuiMT2 consists of 108 bp ORF coding for 35 amino acids with a predicted molecular weight of $3.5 \mathrm{kDa}$ and $\mathrm{pI} 4.14$. Both the sequences were rich in cysteine $(20.5 \%$ cysteine) and had no aromatic amino acids.

Expression analysis of both SiMT genes using qPCR: The impact of $\mathrm{Cu}$ on the induction of both SuiMT1 and SuiMT2 genes was studied using quantitative real time PCR analysis. The mRNA accumulation of both the genes increased rapidly when exposed to increasing concentrations of $\mathrm{Cu}$. At an initial stress of $100 \mu \mathrm{M}$, the expression of SuiMT1 was induced 1.5 times whereas SuiMT2 was induced 3 times of the control. Further, on increasing the $\mathrm{Cu}$ concentration to $400 \mu \mathrm{M}$ there was increased SuiMT1 mRNA accumulation by $\sim 7$ folds and that of SuiMT2 by $\sim 13$ folds (Fig. 3). However, the expression of reference gene actin remained unaltered. This shows that the expression of SuiMT2 is more induced under $\mathrm{Cu}$ stress than SuiMT1.

Functional complementation in yeast mutants: The role of both SuiMT1 and SuiMT2 in Cu tolerance was validated by their functional complementation into the Saccharomyces cerevisiae mutant cupl $^{\Delta}$ (sensitive to copper) and wild type DTY3. Both genes were ligated into pFL61 vector and transformed into cupl $^{4}$ and DTY3. The growth of transformants was then monitored on SD-ura medium supplemented with and without $\mathrm{Cu}(150 \mu \mathrm{M})$. Drop test analysis revealed that the transformants carrying SuiMT1 and SuiMT2 effectively restored the $\mathrm{Cu}$ tolerance in yeast mutant cup $1^{4}$, whereas the same mutant when transformed with only pFL61 could not survive (Fig. 4a).

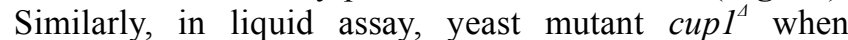
transformed with pFL61 could not grow at $\mathrm{Cu}-150 \mu \mathrm{M}$, whereas the transformants carrying pFL61+SuiMT1 and pFL61+SuiMT2successfully restored the $\mathrm{Cu}$ tolerance in cup $1^{4}$ (Fig. 4b). The wild type DTY3 was used as a positive control.

\section{DISCUSSION}

ECM fungi are very well known for their potential role in protecting the host plant from heavy metal stress (Colpaert et al., 2011; Khullar and Reddy, 2018, 2019a, b). A new ECM species Suillus indicus has been recently isolated from the conifer forest of northwestern Himalayas, India (Verma and Reddy, 2015). The present study focuses on identifying the response of $S$. indicus to $\mathrm{Cu}$ stress and the detoxification mechanisms involved in it. When exposed to increasing concentrations of $\mathrm{Cu}$, the growth of $S$. indicus abated. The half minimum inhibitory concentration was recorded at $\sim 170 \mu \mathrm{M}$

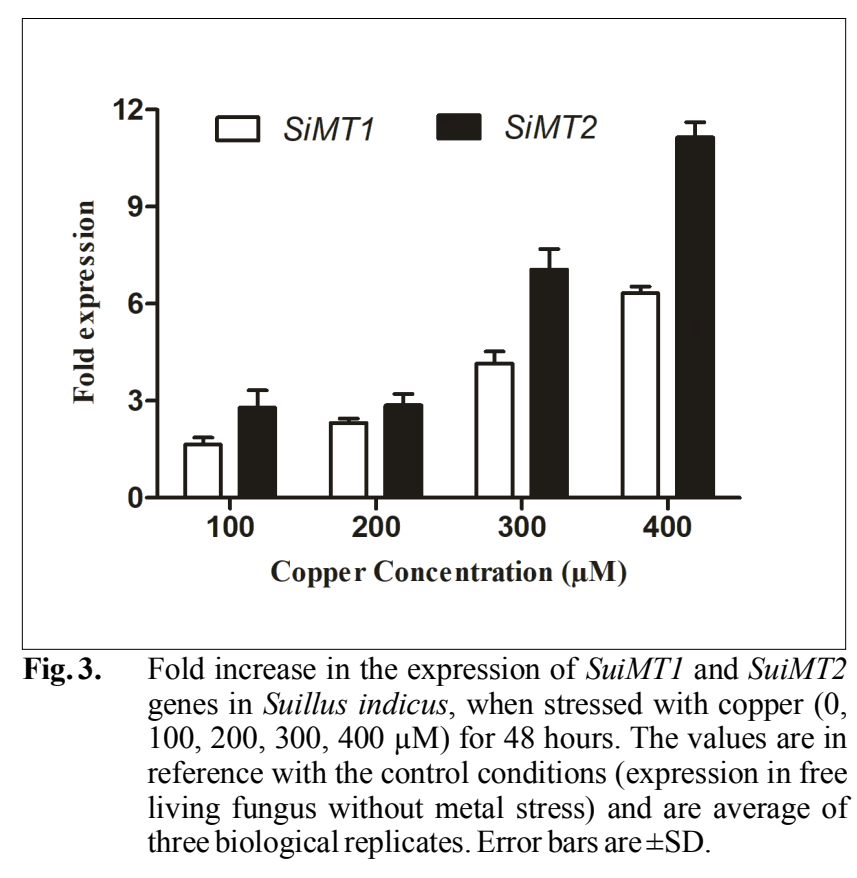




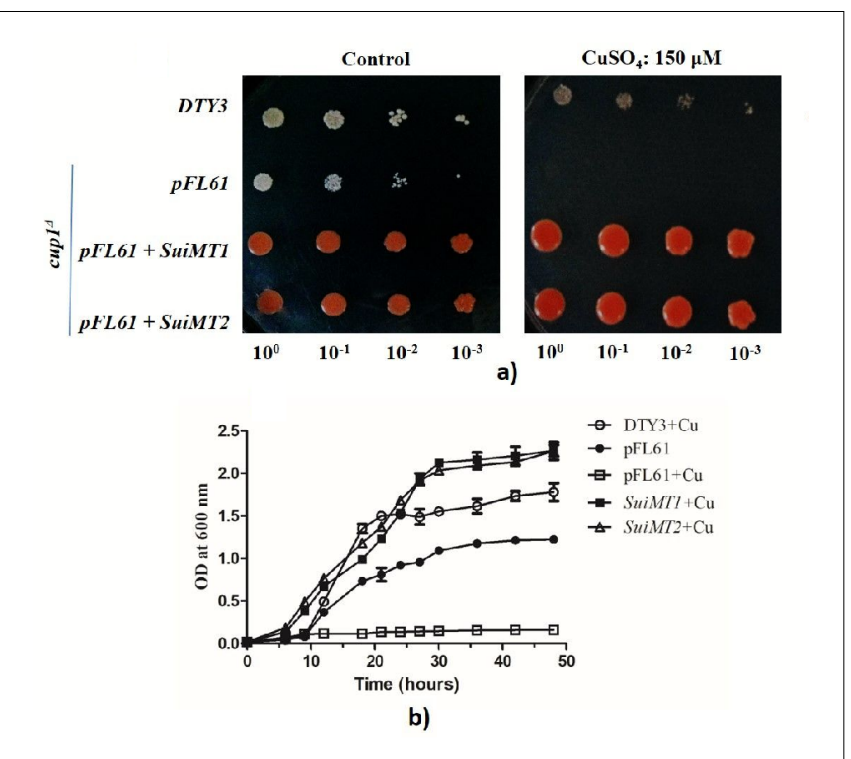

Fig. 4. Functional complementation of SuiMT1 and SuiMT2 genes in $S$. cerevisiae mutant cup $^{\Delta}{ }^{\Delta}$ by a) drop test and b) liquid broth assay on SD-ura medium supplemented with and without $150 \mu \mathrm{MCuSO}_{4}$. The wild type DTY3 was used as the positive control.

which is almost half of the $\mathrm{IC}_{50}$ value reported for $S$. himalayensis $(322 \mu \mathrm{M})$ (Kalsotra et al., 2018). The Cu uptake tendencies of $S$. indicus were also comparable with that of $S$. himalayensis.

Two MT genes SuiMT1 and SuiMT2 were identified from $S$. indicus cDNA using the primers designed from EST transcripts of S. luteus. Both the genes showed homology with the already reported MTs from various basidiomycetes such as Amanita strobiliformis (AsMT2; AG004615), Serendipita indica (SiMT1; ACT83730), Laccaria bicolor (LbMT1; AHI43933); Russula atropurpurea (RaMT1; AHA31882), Fomitopsis rosea (FrMT1; TFY60696) and Coprinopsis cinerea (CcMT1; XP001833429) (Leonhardt et al., 2014; Reddy et al., 2014; Hložková et al., 2016). Binz and Kägi classified MTs into 15 families based on their length and primary structure of sequences (Binz and Kägi, 1999). The length of the MTs varied from 24 amino acids to 257 amino acids. Both SuiMT1 and SuiMT2 genes belong to the family 8 in the above-mentioned classification.

Copper and zinc are the two most potential inducers of metallothioneins in ectomycorrhizal systems (Palacios et al., 2011; Khullar and Reddy, 2018). The intracellular accumulation of metal ions triggers the rapid transcriptional induction of MTs, which in turn sequester metal ions, thus minimizing their toxicity. $\mathrm{Cu}$ - induced metallothioneins have been reported in various ECM fungi like Pisolithus albus (PaMT1) (Reddy et al., 2016), Laccaria bicolor (LbMT1 and LbMT2) (Reddy et al., 2014), Amanita strobiliformis (AsMT1c) (Osobova et al., 2011), Hebeloma cylindrosporum (HcMT1) (Ramesh et al., 2009), Paxillus involutus (PiMT) (Bellion et al., 2007), Suillus luteus (SlMT1 and SlMT2) (Nguyen et al., 2017), and Suillus himalayensis (ShMT1 and ShMT2) (Kalsotra et al., 2018). MTs are induced by the same metal ions that bind to the MT proteins, leading to the direct activation of the defense mechanisms (Waalkes and Goering, 1990; Khullar and Reddy, 2018). Both SuiMT1 and SuiMT2 are actively induced under $\mathrm{Cu}$ stress, thus they can be defined as potential $\mathrm{Cu}$-thioneins. Since different MTs respond differently in the same organism (Khullar and Reddy, 2018), in S. indicus, SuiMT2 has been more rapidly induced than SuiMT1 under $\mathrm{Cu}$ stress. Many studies have reported the presence of C-X-C, C-X-X-C or C-X-Y-C motifs in proteins primary sequence as the main characteristic feature of metallothionein. The primary protein sequence of L. bicolor MT1, A. strobiliformis MT1 and MT2, P. involutus MT1, P. albus MT1, $S$. himalayensis MT1 and MT2 had three conserved C-X-C motifs where as L. bicolor MT2, H. cylindrosporum MT2, H. mesophaeum MT2 and MT3 had six conserved C-X-C motifs and S. luteus MTa and MTb, A. stobiliformis MT3 had five C-X-C motifs with one C-X-Y-C motif conserved (Bellion et al., 2007; Ramesh et al., 2009; Reddy et al., 2014; Sácký et al., 2014; Hložková et al.,2016; Reddy et al., 2016; Nguyen et al., 2017; Kalsotra et al., 2018). In both SuiMT1 and SuiMT2, three conserved C-X-C motifs were found.

The function of both SuiMT1 and SuiMT2 in Cudetoxification was validated using a functional

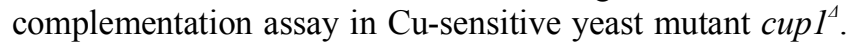
Transformation of the yeast mutant with SuiMT1 and SuiMT2 under the control of phosphoglycerokinase (PGK) promoter successfully restored the $\mathrm{Cu}$ tolerance capability of $S$.

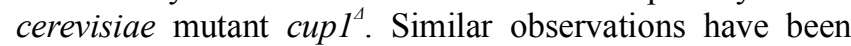
reported in L. bicolor, H. cylindrosporum, $S$. himalayensis, $S$. luteus, P. albus and P. involutus, where the yeast mutant when transformed with the fungal MT gene successfully restored its metal tolerance ability (Bellion et al., 2007; Ramesh et al., 2009; Reddy et al., 2014; Reddy et al., 2016; Nguyen et al., 2017). Thus, it can be concluded that the MTs- SuiMT1 and SuiMT2, contribute to $\mathrm{Cu}$ homeostasis and detoxification in ectomycorrhizal fungus $S$. indicus and may be responsible for protecting the host plant under the $\mathrm{Cu}$ stress. The present study provided insight into the mechanisms involved in $\mathrm{Cu}$ detoxification in ectomycorrhizal fungus $S$. indicus. This understanding can further help in using fungus as the model organism for studying $\mathrm{Cu}$ detoxification mechanism or in developing various bioremediation technologies.

\section{CONCLUSION}

The present study provides insight into the molecular mechanism involved in copper detoxification in ECM fungus $S$. indicus. The characterization of two genes SuiMT1 and SuiMT2 highlighting their potential role in complementing

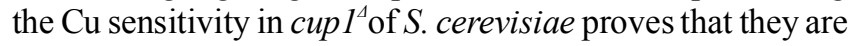
$\mathrm{Cu}$-thioneins and could play an important role in $\mathrm{Cu}$ homeostasis in S. indicus.

\section{ACKNOWLEDGEMENT}

This work was funded by the project grant BT/PR8339/BCE/ 8/1045/2013 from Department of Biotechnology, Ministry of Science and Technology, Govt. of India, India. Authors are thankful to Thapar University, Patiala, India for the laboratory assistance. 


\section{REFERENCES}

Atrián-Blasco, E. et al. 2017. Chemistry of mammalian metallothioneins and their interaction with amyloidogenic peptides and proteins. Chem. Soc. Rev. 46: 7683-7693.

Bano, A. et al. 2018. Biosorption of heavy metals by obligate halophilic fungi. Chemosphere 199: 218-222.

Bellion, M. et al. 2006. Extracellular and cellular mechanisms sustaining metal tolerance in ectomycorrhizal fungi. FEMS. Microbiol. Lett. 254: 173-181.

Bellion, M. et al. 2007. Metal induction of a Paxillus involutus metallothione in and its heterologous expression in Hebeloma cylindrosporum. New. Phytol. 174: 151-158.

Benes, V. et al. 2018. Two P1B-1-ATPases of Amanita strobiliformis with distinct properties in $\mathrm{Cu} / \mathrm{Ag}$ transport. Front. Microbiol.9: 747.

Binz, P.A. and Kägi, J.H.R. 1999. Metallothionein: Molecular evolution and classification. In: Metallothionein IV. Advances in Life Sciences. (Ed.: Klaassen, C.D.): 7-13, Birkhäuser, Basel.

Calvo, J., Jung, H. and Meloni, G. 2017. Copper metallothioneins. IUBMB. Life 69: 236-245.

Colpaert, J.V. et al. 2011. How metal-tolerant ecotypes of ectomycorrhizal fungi protect plants from heavy metal pollution. Ann. For. Sci. 68: 17-24.

Drozd, A. et al. 2018. Crosstalk of the structural and zinc buffering properties of mammalian metallothionein2. Metallomics 10: 595-613.

Hamer, D.H., Thiele, D.J. and Lemontt, J.E. 1985. Function and autoregulation of yeast copperthionein. Science 228: $685-690$.

Heid, C.A. et al. 1996. Real time quantitative PCR. Genome. Res. 6: 986-994.

Hložková, K. et al. 2016. Characterization of three distinct metallothionein genes of the Ag-hyperaccumulating ectomycorrhizal fungus Amanita strobiliformis. Fungal. biol. 120: 358-369.

Huang, Y.Y. et al. 2018. Cloning, characterization and expression analysis of metallothioneins from Ipomoe aquatica and their cultivar-dependent roles in $\mathrm{Cd}$ accumulation and detoxification. Ecotoxicol. Environ. Saf. 165: 450-458.

Imam, H.T. and Blindauer, C.A. 2018. Differential reactivity of closely related zinc (II)-binding metallothioneins from the plant Arabidopsis thaliana. J. Biol. Inorg. Chem. 23: 137-154.

Iturbe-Espinoza, P. et al. 2016. The fungus Tremella mesenterica encodes the longest metallothionein currently known: gene, protein and metal binding characterization. PloS. One 11: p.e0148651.
Kalsotra, T. et al. 2018. Metal induction of two metallothionein genes in the ectomycorrhizal fungus Suillus himalayensis and their role in metal tolerance. Microbiol. 164: 868-876.

Khullar, S. and Reddy, M.S. 2018. Ectomycorrhizal fungi and its role in metal homeostasis through metallothionein and glutathione mechanisms. Curr. Biotechnol. 7: 231-241.

Khullar, S. and Reddy, M.S. 2019a. Cadmium induced glutathione bioaccumulation mediated by $\gamma$ glutamylcysteine synthetase in ectomycorrhizal fungus Hebeloma cylindrosporum BioMetals 32: 101-110.

Khullar, S. and Reddy, M.S. 2019b. Cadmium and arsenic responses in the ectomycorrhizal fungus Laccaria bicolor: glutathione metabolism and its role in metal (loid) homeostasis. Environ. Microbiol. Rep. 11: 5361.

Leonhardt, T. et al. 2014. Metallothionein-like peptides involved in sequestration of $\mathrm{Zn}$ in the $\mathrm{Zn}$ accumulating ectomycorrhizal fungus Russula atropurpurea. Metallomics. 6: 1693-1701.

Li, S. et al. 2016. The induction of metallothioneins during pulsed cadmium exposure to Daphnia magna: recovery and trans-generational effect. Ecotoxicol. Environ. Saf. 126: 71-77.

Livak, K.J. and Schmittgen, T.D. 2001. Analysis of relative gene expression data using real-time quantitative PCR and the 2- $\Delta \Delta \mathrm{CT}$ method. Methods 25: 402408.

Nguyen, H. et al. 2017. A novel, highly conserved metallothionein family in basidiomycete fungi and characterization of two representative SIMTa and SlMTb genes in the ectomycorrhizal fungus Suillus luteus. Environ. Microbiol. 19: 2577-2587.

Osobová, M. et al. 2011. Three metallothionein isoforms and sequestration of intracellular silver in the hyperaccumulator Amanita strobiliformis. New. Phytol. 190: 916-926.

Palacios, Ò., Atrian, S. and Capdevila, M. 2011. Zn-and Cuthioneins: a functional classification for metallothioneins? J. Biol. Inorg. Chem. 16: 991.

Ramesh, G. et al. 2009. Different patterns of regulation for the copper and cadmium metallothioneins of the ectomycorrhizal fungus Hebeloma cylindrosporum. Appl. Environ. Microbiol. 75: 2266-2274.

Reddy, M.S. et al. 2016. Metal induction of a Pisolithus albus metallothionein and its potential involvement in heavy metal tolerance during mycorrhizal symbiosis. Environ. Microbiol. 18: 2446-2454.

Reddy, M.S. et al. 2014. Differential expression of metallothioneins in response to heavy metals and their involvement in metal tolerance in the symbiotic basidiomycete Laccaria bicolor. 
Microbiol. 160: 2235-2242.

Ruytinx, J. et al. 2011. Transcriptome analysis by cDNAAFLP of Suillus luteus Cd-tolerant and Cd-sensitive isolates. Mycorrhiza 21: 145-154.

Ruytinx, J. et al. 2013. Zinc export results in adaptive zinc tolerance in the ectomycorrhizal basidiomycete Suillus bovinus. Metallomics. 5: 1225-1233.

Sácký, J. et al. 2014. Intracellular sequestration of zinc, cadmium and silver in Hebeloma mesophaeum and characterization of its metallothionein genes. Fungal. Genet. Biol. 67: 3-14.

Sacký, J., Leonhardt, T. and Kotrba, P. 2016. Functional analysis of two genes coding for distinct cation diffusion facilitators of the ectomycorrhizal $\mathrm{Zn}$ accumulating fungus Russula atropurpurea. BioMetals. 29: 349-363.

Sambrook, J., and Russell, D.W. 2001. Molecular Cloning: A Laboratory Manual. Cold Spring Harbor, NY, USA: Cold Spring Harbor Laboratory Press.

Singh, M. et al. 2010. Plasmid DNA transformation in Escherichia coli: effect of heat shock temperature, duration, and cold incubation of $\mathrm{CaCl}_{2}$ treated cells. IOSR. J. Biotechnol. Biochem. 6: 561-568.

Solioz, M. 2018. Copper Homeostasis in Gram-Positive Bacteria. In: Copper and Bacteria. (Ed.: Solioz, M.) Springer Briefs in Molecular Science: 21-48 Springer, Cham.

Stearns, T., Ma, H. and Botstein, D. 1990. Manipulating yeast genome using plasmid vectors. In: Methods in enzymology. (Ed.: Goeddel, D.V.). 185: 280-297. Academic Press.

Verma, B. and Reddy, M.S. 2015. Suillus indicus sp. Nov. (Boletales, Basidiomycota), a new boletoid fungus from north western Himalayas, India. Mycology 6: $35-41$.

Waalkes, M.P. and Goering, P.L. 1990. Metallothionein and other cadmium-binding proteins: recent developments. Chem. Res. Toxicol. 3: 281-288.

Zahid, M.T. et al. 2016. Molecular characterization of a copper metallothionein gene from a ciliate Tetrahymena farahensis. J. Cell. Biochem. 117: 1843-1854. 